\title{
Der Feind in meinem Beet
}

\author{
Neues aid-Heft „Schadschnecken - Biologie, Arten und Bekämpfung“
}

Online publiziert: 25. September 2014

(C) Springer-Verlag Berlin Heidelberg 2014

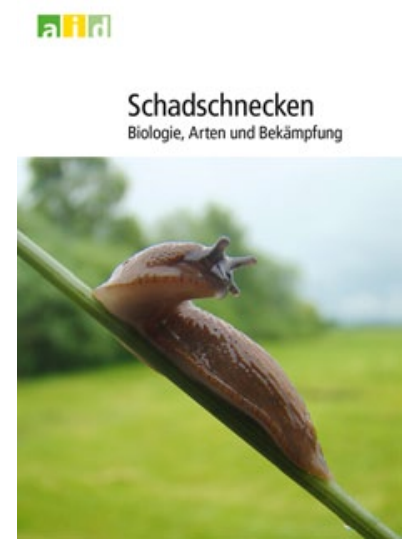

Ausgehöhlte Früchte, großflächige Fraßstellen an Blättern und verdächtige Schleimspuren, die sich kreuz und quer durchs Gemüsebeet ziehen - das ist der Albtraum aller Hobbygärtner. Denn wer schon einmal versucht hat, im eigenen Garten Gemüse anzubauen, weiß sofort, wer hinter diesen Spuren steckt: Schnecken. Doch Schnecke ist nicht gleich Schnecke. Nicht jede Art frisst sich über Nacht durch alle Salatköpfe und nagt nebenbei noch die Erdbeeren an. Aber wie unterscheidet man als Laie die schädlichen von unschädlichen Arten? Wie leben und vermehren sich Schnecken eigentlich? Und vor allem: Wie kann man sie möglichst ohne Gift vom Gemüsebeet fernhalten? Antworten auf diese und viele weitere Fragen rund um den gefürchteten und zugleich faszinierenden Schädling gibt das neu überarbeitete aid-Heft „Schadschnecken“. Der Leser erfährt, welche Rolle Schnecken im Ökosystem spielen, wie sie sich ernähren und fortpflanzen und wie sich daraus Ansatzpunkte für die Bekämpfung ableiten lassen. Ausführlich werden die häufigsten Schadschneckenarten beschrieben und Merkmale zur Unterscheidung von unschädlichen Arten dargestellt. Die Liste reicht von der Spanischen Weg- schnecke über den Gewächshausschnegel bis zur Weinbergschnecke. Viele hochwertige Bilder helfen dabei, die Tiere zu identifizieren. Auch wie man natürliche Schneckenfeinde wie Igel, Glühwürmchen und Hornfliegen fördern kann, um die Schneckenplage einzudämmen, wird erläutert. Schwerpunkt des Heftes sind bewährte Strategien, mit denen sich Schnecken im Hausgarten, aber auch im professionellen Gemüse- und Ackerbau in Schach halten lassen. Dazu gehören vorbeugende Maßnahmen wie resistente Pflanzenarten oder mechanische Barrieren, aber auch direkte Bekämpfungsmöglichkeiten wie der Einsatz von Nematoden, das Anlocken und Absammeln der Tiere und chemische Mittel. Zudem wird gezeigt, wie sich die Verfahren sinnvoll kombinieren lassen. Damit gibt das Heft einen spannenden Einblick in die Lebensweise einer ungewöhnlichen Tiergruppe und Hobbygärtnern, Acker- und Gemüsebauern praxisnahe Tipps für eine nachhaltige Bekämpfung. aid-Heft „Schadschnecken - Biologie, Arten und Bekämpfung", 64 Seiten, 2. Auflage 2014

Best.-Nr.: 1509, ISBN/EAN: 978-3-8308-1144-2, Preis: 2,50 $€$, zzgl. einer Versandkostenpauschale von $3,00 €$ aid infodienst e. V., Heilsbachstraße 16, 53123 Bonn Telefon: 038204 66544*, Telefax: 022884 99-200

*Kosten: 9 Cent pro Minute aus dem deutschen Festnetz, Anrufe aus dem Mobilfunknetz maximal 42 Cent pro Minute. Aus dem Ausland können die Kosten abweichen.

E-Mail: Bestellung@aid.de, Internet: www.aid-medienshop.de 